\title{
Non-local Operations: Purification, storage, compression, tomography, and probabilistic implementation
}

\author{
W. Dür and J. I. Cirac \\ Institut für Theoretische Physik, Universität Innsbruck,A-6020 Innsbruck, Austria
}

(October 24, 2018)

\begin{abstract}
We provide several applications of a previously introduced isomorphism between physical operations acting on two systems and entangled states [1]. We show: (i) how to implement (weakly) non-local two qubit unitary operations with a small amount of entanglement; (ii) that a known, noisy, non-local unitary operation as well as an unknown, noisy, local unitary operation can be purified; (iii) how to perform the tomography of arbitrary, unknown, non-local operations; (iv) that a set of local unitary operations as well as a set of non-local unitary operations can be stored and compressed; (v) how to implement probabilistically two-qubit gates for photons. We also show how to compress a set of bipartite entangled states locally, as well as how to implement certain non-local measurements using a small amount of entanglement. Finally, we generalize some of our results to multiparty systems.
\end{abstract}

03.67.-a, 03.65.Bz, 03.65.Ca, 03.67.Hk

\section{INTRODUCTION}

In recent years, much of the theoretical effort in quantum information (QI) theory was focused on establishing properties of states and techniques to manipulate them. One of the main purposes was - and is - the characterization and quantification of entanglement properties of multiparticle states, as entangled states play an important role in several applications of QI. Many schemes and applications which involve the manipulation of quantum states were discovered. Among them, we have teleportation [2], purification of noisy entanglement [3 5], quantum data compression [6], quantum cloning [7], and quantum tomography [8].

In practice, entangled states are created by some physical action. This suggest that establishing properties of operations may play an important role in QI as well. First steps in this direction have been recently reported [9-12]. In particular, in Ref. [10 the entanglement capability of interaction Hamiltonians between two systems has been introduced and analyzed. This quantity measures the maximum rate at which entanglement can be produced given some particular interaction. On the other hand, the entanglement cost for the implementation of non-local operations was also considered recently 133 16]; in particular, several examples, all dealing with an integer number of ebits required for the implementation of certain non-local operations, were introduced.

In Ref. [1] we introduced an isomorphism which relates physical operations (completely positive maps $(\mathrm{CPM}) \mathcal{E}$ ) and states (positive operators $E$ ). This isomorphism turns out to be an important ingredient in the understanding of entanglement properties of operations in general. In this paper, we will first review the results obtained in Ref. 迎:

- In order to study the separability and entangling properties of operations $\mathcal{E}$, it suffices to study the separability properties of the associated operators $E$ [1]. In particular, one can use all the results obtained for the separability of states [17]. This allows to answer questions like "Given a CPM $\mathcal{E}$, can it be used to create entanglement?". Such questions may be relevant in experiments, where one might want to know whether a certain machine (set up) can be used to create entangled states.

- One can easily construct physical operations $\mathcal{E}$ which can generate bound entangled states (BES).

- An important problem in the context of distributed quantum computation 18 is the implementation of non-local unitary operations. In [1], it was shown that an arbitrary 2-qubit unitary operation can be implemented using an amount of entanglement which is proportional to the entanglement capability of the operation 9,10 .

Then, we will discuss several other applications of the isomorphism:

- One can perform two-qubit gates probabilistically in the context of single photon experiments via creation of entangled states assisted by incomplete Bell measurements.

- Several techniques concerning quantum statese.g. quantum teleportation [2], quantum state purification [3, 4, quantum data compression [6] and quantum cloning [7]- were consider in recent years. The isomorphism allows in a simple way to obtain similar results for operations. That is, noisy unitary operations can be purified, and sets of them can be stored and compressed. Furthermore, it is possible to clone unitary operations as well as to teleport them [19,20]. Finally, one can easily see how to perform the tomography [21,22] of general non-local operations locally.

- One can perform certain non-local measurements by using a small amount of entanglement. 
This paper is organized as follows. In Sec. [I], the isomorphism between operations and states is reviewed and several implications are discussed. This isomorphism provides the basic tool for a number of applications which are presented in the preceding sections. In Sec III, we show how to implement non-local two-qubit unitary operations with unit probability, consuming an amount of entanglement which is proportional to the entanglement capability of the operation. Sec. IV is concerned with purification of noisy operations, while Sec. V deals with tomography of arbitrary non-local operations. In Sec. VI it is shown how to implement probabilistically two-qubit operations in the context of single photon experiments. Next, in Sec. VII, storage and compression of non-local unitary operations are discussed, while Sec. VIII is concerned with the implementation of non-local measurements. Finally, in Sec. IX the isomorphism is extended to multi-party systems. We summarize our results in Sec. X.

\section{ISOMORPHISM BETWEEN OPERATIONS AND STATES}

In [1], an isomorphism which relates physical operations [equivalently completely positive maps (CPM) $\mathcal{E}$ ] acting on two systems and (unnormalized) states (positive operators $E$ ) was introduced. This isomorphism is an extension of the one introduced by Jamiolkowski [23]. To be specific, let us consider two spatially separated parties $A$ and $B$, each of them possessing several particles Let $S=\{|i\rangle\}_{i=1}^{d}$ be an orthonormal basis, and

$$
\begin{aligned}
|\Phi\rangle_{A_{1,2}} & =\frac{1}{\sqrt{d}} \sum_{i=1}^{d}|i\rangle_{A_{1}} \otimes|i\rangle_{A_{2}}, \\
P_{A_{1,2}} & =|\Phi\rangle_{A_{1,2}}\langle\Phi|,
\end{aligned}
$$

where $|\Phi\rangle$ is a maximally entangled state (MES) and $P$ a projector on this state. We consider a CPM $\mathcal{E}$ acting on the density operator of two $d$-level systems, one belonging to $A$ and one to $B$. Then, there exist an isomorphism (linear one to one correspondence) between the CPM $\mathcal{E}$ and a positive operator $E$ [1] defined by the following relations:

$$
\begin{aligned}
E_{A_{1,2}, B_{1,2}} & =\mathcal{E}\left(P_{A_{1,2}} \otimes P_{B_{1,2}}\right) \\
\mathcal{E}\left(\rho_{A_{1} B_{1}}\right) & =d^{4} \operatorname{tr}_{A_{2,3} B_{2,3}}\left(E_{A_{1,2}, B_{1,2}} \rho_{A_{3} B_{3}} P_{A_{2,3}} P_{B_{2,3}}\right) .
\end{aligned}
$$

These equations have a very simple interpretation: On the one hand, Eq. (3a) states that given a $\mathrm{CPM} \mathcal{E}$, one

\footnotetext{
${ }^{1}$ We will denote particles belonging to party $A$ by $A_{1}, A_{2}, A_{3}$, where each of the particles is a $d$-level systems. We will also use the notation $A_{1,2}$ to refer to particles $A_{1}, A_{2}$. A similar notation is used for particles belonging to party $B$.
}

can always produce the state $E$ associated with $\mathcal{E}$ by applying $\mathcal{E}$ to particles $A_{1} B_{1}$ if they are prepared in the state $\tilde{P}=P_{A_{1,2}} \otimes P_{B_{1,2}}$. Note that $\tilde{P}$ is a product state with respect to parties $A$ and $B$, while it is a local MES in the system belonging to party $A$ and $B$ respectively. On the other hand, Eq. (3b) says that given the state $E$ (of particles $A_{1,2}, B_{1,2}$ ), one can implement the operation $\mathcal{E}$ on an arbitrary state $\rho$ of two $d$-level systems (particles $\left.A_{3} B_{3}\right)$ by measuring the projector $P$ locally in $A_{2,3}$ and $B_{2,3}$. After a successful measurement - where the probability of success is given by $p=1 / d^{4}$ - particles $A_{1} B_{1}$ are found in the state $\mathcal{E}(\rho)$. In summary, a CPM $\mathcal{E}$ can be used to prepare a state $E$, which in turn can be used to implement $\mathcal{E}$ with a certain probability of success.

\section{IMPLEMENTATION OF NON-LOCAL UNITARY OPERATION WITH UNIT PROBABILITY}

In [1], it was shown how to implement an arbitrary non-local two-qubit unitary operation with arbitrary high accuracy and unit probability, consuming an amount of entanglement which is proportional to the entangling capability of the operation. Here, we review and improve this procedure. To this aim, —as in Ref. [1] we consider a family of phase gates

$$
U\left(\alpha_{N}\right) \equiv e^{-i \alpha_{N} \sigma_{x}^{A_{1}} \otimes \sigma_{x}^{B_{1}}}, \alpha_{N} \equiv \pi / 2^{N}
$$

where the $\sigma$ 's are Pauli matrices. We show that:

(i) The operation $U\left(\alpha_{N}\right)$ can be implemented with probability $p=1 / 2$.

(ii) By applying a finite sequence of operations of the form (4), each being implemented with probability $p=1 / 2$ using (i), one can achieve that the operation $U\left(\alpha_{N}\right)$ is applied with probability $p=1$.

(iii) Using gates of the form (4) with binary angles $\alpha_{N}=\pi / 2^{N}$, one can implement phase gates with arbitrary angle $\alpha$.

(iv) An arbitrary two-qubit unitary operation can be implemented using a sequence of three operations of the form $U(\alpha)$, assisted by local unitary transformations.

While (i-iii) are already explained in [1], the implementation of (iv) is different to the implementation described in 1]. There, an infinite sequence of operations of the form $U(\alpha)$ was required in order to implement an arbitrary two-qubit operation, while here a finite sequence consisting of three operations suffices. The required amount of entanglement is also smaller using the new method.

Since steps (i-iii) will be crucial for the understanding of some procedures described in later sections, we 
discuss them again in detail. We start out by showing (i). First we note that the operator associated with the unitary operation $U\left(\alpha_{N}\right)$ (4) is given by $E_{A_{1,2}, B_{1,2}}=$ $\left|\psi_{\alpha_{N}}\right\rangle_{A_{1,2}, B_{1,2}}\left\langle\psi_{\alpha_{N}}\right|$, where

$$
\begin{aligned}
\left|\psi_{\alpha_{N}}\right\rangle_{A_{1,2}, B_{1,2}}= & \cos \left(\alpha_{N}\right)\left|\Phi^{+}\right\rangle_{A_{1,2}}\left|\Phi^{+}\right\rangle_{B_{1,2}} \\
& -i \sin \left(\alpha_{N}\right)\left|\Psi^{+}\right\rangle_{A_{1,2}}\left|\Psi^{+}\right\rangle_{B_{1,2}}
\end{aligned}
$$

and $\left|\Phi^{+}\right\rangle=(|00\rangle+|11\rangle) / \sqrt{2},\left|\Psi^{+}\right\rangle=\mathbb{1} \otimes \sigma_{x}\left|\Phi^{+}\right\rangle=(|01\rangle+$ $|10\rangle) / \sqrt{2}$ are Bell states. In general, the Bell basis is defined as

$$
\left|\Psi_{i_{1}, i_{2}}\right\rangle=\mathbb{1} \otimes \sigma_{i_{1}, i_{2}}\left|\Phi^{+}\right\rangle,
$$

where $\sigma_{1,1}=\mathbb{1}, \sigma_{1,2}=\sigma_{x}, \sigma_{2,1}=\sigma_{y}, \sigma_{2,2}=\sigma_{z}$ and $\left|\Psi_{1,1}\right\rangle=\left|\Phi^{+}\right\rangle,\left|\Psi_{1,2}\right\rangle=\left|\Psi^{+}\right\rangle,\left|\Psi_{2,1}\right\rangle=i\left|\Psi^{-}\right\rangle,\left|\Psi_{2,2}\right\rangle=$ $\left|\Phi^{-}\right\rangle$are MES. Note that for convenience - to ensure a simple notation below as well as in the remaining sections-, we use a redundant definition of the Bell basis. We consider a situation similar to the one described in (3b); that is, particles $A_{1,2} B_{1,2}$ are prepared in state (5), and $\rho_{A_{3} B_{3}}$ is the state on which a CPM $\mathcal{E}$ - in our case, the unitary operation $U\left(\alpha_{N}\right)$ (4) - should be applied. Now, a Bell measurement is performed on particles $A_{2,3}\left[B_{2,3}\right]$. Assume that the result associated to $\left|\Psi_{i_{1}, i_{2}}\right\rangle$ $\left[\left|\Psi_{j_{1}, j_{2}}\right\rangle\right]$ is obtained. In this case, the state of system $A_{1} B_{1}$ is proportional to

$$
\mathcal{E}\left(\left(\sigma_{i_{1}, i_{2}}^{A_{1}} \otimes \sigma_{j_{1}, j_{2}}^{B_{1}}\right) \rho_{A_{1} B_{1}}\left(\sigma_{i_{1}, i_{2}}^{A_{1}} \otimes \sigma_{j_{1}, j_{2}}^{B_{1}}\right)\right) .
$$

Thus, as a result of the measurement we either implement the $\mathrm{CPM} \mathcal{E}$, or some unitary operation followed by the CPM. We now proceed as follows: In case the result of the measurement was $i_{1}, i_{2}\left[j_{1}, j_{2}\right]$, the local unitary operation $\sigma_{i_{1}, i_{2}}\left[\sigma_{j_{1}, j_{2}}\right]$ is applied on $A_{1}\left[B_{1}\right]$. In case that $\mathcal{E}$ is given by the unitary operation $U\left(\alpha_{N}\right)$ (画), one readily observes that the resulting operation performed on $\rho_{A_{1} B_{2}}$ after this procedure will be (i) $U\left(\alpha_{N}\right)$ if $i_{1}=j_{1}$; (ii) $U\left(\alpha_{N}\right)^{\dagger}=U\left(-\alpha_{N}\right)$ if $i_{1} \neq j_{1}$. Due to the fact that all measurement outcomes are equal probable, we have that with probability $p=1 / 2$ the desired operation $U\left(\alpha_{N}\right)$ was applied, while with $p=1 / 2$ the operation $U\left(-\alpha_{N}\right)$ was performed, from which (i) follows.

Before we proceed, we investigate the amount of nonlocal entanglement (between systems $A$ and $B$ ) which is required to perform the described procedure. The amount of entanglement of the state $\left|\psi_{\alpha_{N}}\right\rangle$ (5) is given by its entropy of entanglement

$$
E\left(\psi_{\alpha_{N}}\right)=-x_{N} \log _{2}\left(x_{N}\right)-\left(1-x_{N}\right) \log _{2}\left(1-x_{N}\right),
$$

where $x_{N}=\cos ^{2}\left(\alpha_{N}\right)=\cos ^{2}\left(\pi / 2^{N}\right)$. That is, the amount of entanglement required to implement the operation $U\left(\alpha_{N}\right)$ with probability $p=1 / 2$ is given by (8). We have that $U(\pi / 2)=-i \sigma_{x} \otimes \sigma_{x}$ is a local gate and thus $E\left(\psi_{\alpha_{1}}\right)=0$, while $E\left(\psi_{\alpha_{2}}\right)=1$, i.e. one ebit of entanglement is required. For $N \geq 2$, we have that $E\left(\psi_{\alpha_{N}}\right)$ is monotonically decreasing with $N$. The amount of classical communication is given by one bit in both directions (the value of $i_{1}\left[j_{1}\right]$ respectively has to be transmitted).

Regarding (ii), we have to show how to obtain a probability of success $p=1$ by making use of the procedure described above. Note that with probability $p=1 / 2$, we succeed and apply the desired gate, while with $p=1 / 2$ we fail and apply $U\left(-\alpha_{N}\right)$ instead. Now, if we fail, we repeat the procedure but with systems $A_{1,2} B_{1,2}$ prepared in the state $\left|\psi_{2 \alpha_{N}}\right\rangle$. With a probability $1 / 2$ we succeed, and otherwise we will have applied $U\left(-\alpha_{N}\right)^{3}$ to the original state instead. We continue in the same vain, that is in the $k$-th step we use systems $A_{1,2} B_{1,2}$ prepared in the state $\left|\psi_{2^{k-1} \alpha_{N}}\right\rangle$ so that if we fail altogether we will have applied $U\left(-\alpha_{N}\right)^{2^{k}-1}$. For $k=N$ we have that $U\left(-\alpha_{N}\right)^{2^{N}-1}=-U\left(\alpha_{N}\right)$, and therefore even if we fail we will have applied the right gate, so that the procedure ends. In fact, the $N^{\text {th }}$ step will succeed with $p=1$, as $U(\pi / 2)$ is a local gate which can be implemented with unit probability and without consuming entanglement. That is, a sequence of $N$ operations of the form (4) allows to implement the operation $U\left(\alpha_{N}\right)$ with unit probability, which proves (ii).

Let us investigate the average amount of entanglement which is consumed during this procedure. We have

$$
\bar{E}\left[U\left(\alpha_{N}\right)\right]=\sum_{k=1}^{N}\left(\frac{1}{2}\right)^{k-1} E\left(\psi_{\alpha_{N-k+1}}\right)=\alpha_{N} f_{N},
$$

where

$$
f_{N}=\frac{1}{\pi} \sum_{k=1}^{N} 2^{k} E\left(\psi_{\alpha_{k}}\right)<f_{\infty}=5.97932 .
$$

In (9), the weight factor $p_{k}=(1 / 2)^{k-1}$ gives the probability that the $k$-th step has to be performed. Thus, we obtain $\bar{E}\left[U\left(\alpha_{N}\right)\right]<\alpha_{N} f_{\infty}$; that is, the average amount of entanglement is bounded from above by a quantity which is proportional to the angle $\alpha_{N}$ and thus - for small $\alpha_{N}$ - proportional to the entangling capability of the operation [10]. The average amount of classical communication is given by $2-(1 / 2)^{N-2}$ bits.

To show (iii), we use the fact that any gate $U(\alpha)$ with arbitrary phase $\alpha$ can be approximated with arbitrary high accuracy by a sequence of gates of the form $U\left(\alpha_{N}\right)$. That is, any angle $0 \leq \alpha \leq \pi$ can be written as

$$
\alpha=\pi \sum_{k=1}^{\infty} n_{k} 2^{-k}, n_{k} \in\{0,1\} .
$$

For each $k$, we have that $n_{k}$ is either " 0 " - which means that the rotation $U\left(\alpha_{k}\right)$ does not have to be performedor "1" - which means that the rotation $U\left(\alpha_{k}\right)$ has to be performed-. Operations of the form $U\left(\alpha_{k}\right)$ can be implemented with unit probability using (i-ii). The average amount of entanglement consumed to implement $U(\alpha)$ is bounded by $\bar{E} \leq f_{\infty} \alpha$ ebits. 
Finally, to show (iv), we use the result of Kraus et al. [11. There, it was shown that an arbitrary two-qubit unitary operation can be written in the following form

$$
U_{A B}=V \otimes W e^{-i H} \tilde{V} \otimes \tilde{W},
$$

where $V, W, \tilde{V}, \tilde{W}$ are local operations and

$$
H=\sum_{k=x, y, z}^{3} \mu_{k} \sigma_{k}^{A} \otimes \sigma_{k}^{B} \equiv \sum_{k=1}^{3} H_{k},
$$

where $0 \leq \mu_{k} \leq \pi / 2$. We note that

$$
e^{-i H}=e^{-i H_{1}} e^{-i H_{2}} e^{-i H_{3}},
$$

and $e^{-i H_{k}}$ are - up to a change of local basis - operations of the form (4) for which we already provided a protocol [see (i-iii)]. Using this, we obtain that an arbitrary two-qubit unitary operation can be performed using a sequence of three operations of the form $U(\alpha)$, assisted by local unitary operations, which proves (iv). The required amount of entanglement is bounded by $f_{\infty}\left(\mu_{1}+\mu_{2}+\mu_{3}\right)$ ebits.

\section{PURIFICATION OF NOISY OPERATIONS}

In this Section, we consider purification of a noisy operations. We will discuss two different scenarios:

In the first scenario, we consider two spatially separated parties $A$ and $B$ who want to perform a known, non-local (entangling) unitary operation $U$ between two particles they share. We will assume that $A$ and $B$ are only able to perform the operation $U$ in an imperfect way. So instead of performing $U$ on their particles, they perform some CPM $\mathcal{E}_{U}$. The problem we pose is the following: Given several applications of the noisy operation $\mathcal{E}_{U}$ and arbitrary local resources, can the parties $A$ and $B$ use them to perform the (noiseless) operation $U$ on an arbitrary state of two qubits instead? Under which circumstances is this possible? In case this is possible, we say that the noisy operation is purificable. In Sec. IV A we are going to show when and how it is possible to achieve this task.

The second scenario is concerned with the purification of an unknown, local noisy unitary operation $\mathcal{E}_{U}$, where we explicitly assume a specific form of noise. In Sec. IV B, we provide a procedure to implement an unknown unitary operation perfectly, given several applications of the noisy operation.

In both cases, it turns out that the isomorphism (3) allows to use results obtained for purification of states and thus for a very simple solution to the problem. Regarding the first scenario, the corresponding problem for states is the problem of entanglement distillation of mixed states [4]. For the second scenario, the corresponding problem for states is the purification of a single qubit [24].

\section{A. Purification of a known non-local noisy unitary operation}

We consider two parties $A$ and $B$, who want to perform a joint unitary operation $U$ among two particles they share. For simplicity, let us assume that $U \in S U(4)$, i.e. the particles are qubits. The parties $A$ and $B$ are only capable to perform the operation $U$ in an imperfect way, so they perform some CPM $\mathcal{E}_{U}$ instead. For example, a noisy $N$-qubit operation can be of the form 25

$$
\mathcal{E}_{U}(\rho)=q U \rho U^{\dagger}+\frac{1-q}{2^{N}} \mathbb{1},
$$

i.e. with probability $q$ the desired operation is performed, while with $(1-q)$ a completely depolarized state (described by the identity operator $\mathbb{1}$ ) is produced. The following analysis is not restricted to this specific form of noisy operations.

The operation $U$ is known to both $A$ and $B$. Furthermore, they are allowed to use auxiliary systems and are able to perform all operations (including two-qubit operations) on their individual sites perfectly. In the following, we are going to show that the noisy, entangling operation $\mathcal{E}_{U}$ can be purified if and only if the operator $\rho_{\mathcal{E}}$ corresponding to $\mathcal{E}_{U}$ (3a) is distillable. We also provide a practical protocol to achieve this task.

The purification procedure takes place as follows:

- $\mathcal{E}_{U}$ is used to create several copies of $\rho_{\mathcal{E}}$ (see $(3 \mathrm{a})$ ).

- With help of entanglement distillation for states, out of $\rho_{\mathcal{E}}^{\otimes M}$ a number of MES are created.

- The MES are used to create a set of states of the form (5), either via deterministic state transformation (single copy case) or via entanglement dilution [3.

- Finally, these states are used to implement $U$ with unit probability and arbitrary high accuracy as described in Sec. III.

Now we will show that an operation $\mathcal{E}_{U}$-where $U$ is an entangling operation - is purificable if and only if $\rho_{\mathcal{E}}$ is distillable. This can be seen as follows. On the one hand, if $\rho_{\mathcal{E}}$ is distillable, one can use the procedure described above to purify the noisy operation $\mathcal{E}_{U}$. On the other hand, if $\mathcal{E}_{U}$ is purificable, this implies that the unitary operation $U$ can be performed on an arbitrary state of two qubits, using a sequence of noisy operations $\mathcal{E}_{U}$ assisted by local operations and classical communication. Since $U$ is an entangling operation, the corresponding pure state $E_{U}$ is also entangled. That is, the sequence of operations $\mathcal{E}_{U}$, assisted by local operations and classical communication is capable to create entangled states when acting on a certain separable state. Using the isomorphism (3), we can write this sequence of operations acting on a separable state in terms of a trace over several operators $E_{i}$, where local operations in the sequence 
correspond to separable operators [1]. That is, the only entangled operators which appear in this expression are operators $\rho_{\mathcal{E}}$ corresponding to the noisy operation $\mathcal{E}_{U}$ and the resulting state is entangled. This implies that from several copies of the mixed state $\rho_{\mathcal{E}}$, an entangled pure state can be created. Note that using entanglement distillation for pure states [3], this implies that one can also create a MES. We thus have that $\rho_{\mathcal{E}}$ is distillable, which finishes the proof of our statement.

Since $\mathcal{E}_{U}$ is a general CPM, $\rho_{\mathcal{E}}$ is a mixed state in $C^{4} \otimes \mathbb{C}^{4}$, where no necessary and sufficient condition for distillability is known (see however [27,28). It is known that non-positive partial transposition of $\rho_{\mathcal{E}}$ is a necessary condition for distillability, however there are strong evidences that this condition is not sufficient [27,28]. Using entanglement purification for states, e.g. via the methods discussed in $27,29,4$, one may be able to obtain a MES starting from several copies of $\rho_{\mathcal{E}}$.

Given the error model (15), one can obtain a necessary and sufficient condition for purificability. It turns out that unitary operations which are only weakly entangling (e.g. operations of the form $U(\alpha)$ with $\alpha \ll 1$ ) are much more sensitive to noise than unitary operations which are strongly entangling, e.g. the controlled-not (CNOT) operation 30]. This means that the tolerable error, specified by $(1-q)$ - such that purification of the noisy operation is still possible - in the case of the CNOT is much bigger than for $U(\alpha)$ with $\alpha \ll 1$. For the CNOT and the error model (15), one obtains $q>1 / 9$ in order that purification of the noisy gate be possible 31 . For operations of the form $U(\alpha)$, one finds $q>(16 \cos (\alpha) \sin (\alpha)+1)^{-1}$ as a necessary and sufficient condition that gate purification is possible. For $\alpha=\pi / 2^{13}$, this value is e.g. given by $q>163 / 164 \approx 0.994$, i.e. less than one percent of noise is allowed in this case.

Note that the process of entanglement distillation involves two-qubit joint operations as well. The reason why we treat these (local) operations differently than the (non-local) operation $U$ can be viewed as follows. On the one hand, the parties $A$ and $B$ may be spatially separated and the interaction between the two parties - for example performed through the usage of a (noisy) quantum channel- is much more sensitive to noise than the local operations performed by only one of the parties. On the other hand, each of the parties $A$ and $B$ may be considered to possess a single particle only, each particle containing several levels. Here, the additional levels are used instead of the auxiliary qubits. In this case, the operation $U$ is concerned with the interaction between two different particles, while all local operations (also including multilevel — equivalently multiqubit - operations) are operations performed on a single particle, which are much easier to implement. For example, using atoms or ions with several levels, all local operations can be easily performed [33]. However, controlled interactions between two ions/atoms are very difficult to achieve, which leads to the fact that two-particle gates are noisy while local gates are practically not. Recall that in state purifica- tion, it is similarly assumed that local operations can be performed perfectly and that it has to be known which is the MES which has a large overlap with the mixed state the parties share in order that they can distill this specific MES. Similarly, knowledge of the perfect unitary operation $U$ is required.

One may also consider that the local operations are noisy. In this case, both the process of distillation of states and the implementation of the operation $U$ using several different states and Bell measurements will give rise to some imperfections. The purification of states with imperfect means was studied in 26, 34 and it was found that no MES can be obtained if the local operations are noisy and a certain error model is assumed. However, one is still able to increase the fidelity, i.e. the overlap of the produced state with a MES, where the maximal reachable fidelity is determined by the amount of noise introduced by the local operations. So, instead of producing MES, one produces some mixed state $\rho$. This state $\rho$ may then be transformed to a state which is close to the ones of the form (5). These states can then be used to implement the operation $U$ in an imperfect way, since both the states which are used and the operations which are performed are noisy. Furthermore, one should take into account that a sequence of noisy operations is required in order to implement $U$ with unit probability, so the errors may accumulate. For almost perfect local operations and very noisy non-local operations, one may, however, still expect a purification effect.

\section{B. Purification of an unknown local noisy unitary operation}

Here, we consider a party $A$ who wants to perform a unitary operation $U \in S U(2)$ on a single qubit. The operation cannot be performed perfectly but is subjected to some noise. We will explicitly assume that the imperfect operation $\mathcal{E}_{U}$ is of the form (15] 25] with $N=1$; that is, with probability $q$ the desired unitary operation $U$ is performed, while with probability $(1-q)$ the completely depolarized state $1 / 2 \mathbb{1}$ is produced. Here, in contrast to the previous discussion, we will assume that the operation is local and unknown to $A$ (for example, $\mathcal{E}_{U}$ is provided to $A$ by a second party via a black box). Given that party $A$ is able to perform all operations perfectly, we will show that the unknown noisy unitary operation can be purified, i.e. via several applications of $\mathcal{E}_{U}$, the noiseless operation $U$ can be implemented on an arbitrary qubit. For simplicity, we assume that the unitary operation $U$ is of the form

$$
U(\alpha)=e^{-i \alpha \sigma_{x}}
$$

where $\alpha$ is unknown, however the analysis can be generalized to arbitrary single-qubit unitary operations. The positive operator $E$ corresponding to the imperfect operation $\mathcal{E}_{U}$ is given by 


$$
E=q\left|\Psi_{U}\right\rangle\left\langle\Psi_{U}\right|+\frac{1-q}{4} \mathbb{1}_{4},
$$

where $\left|\Psi_{U}\right\rangle=\cos (\alpha)\left|\Phi^{+}\right\rangle-i \sin (\alpha)\left|\Psi^{+}\right\rangle$.

We proceed as follows. First, we project $E$ on the subspace spanned by $\left\{\left|\Phi^{+}\right\rangle,\left|\Psi^{+}\right\rangle\right\}$and relabel the basis:

$$
|\tilde{0}\rangle=\left|\Phi^{+}\right\rangle,|\tilde{1}\rangle=-i\left|\Psi^{+}\right\rangle .
$$

In case we succeed, which happens with probability $(q+$ 1) $/ 2$, the resulting state will be

$$
\tilde{E}=\lambda\left|\tilde{\Psi}_{U}\right\rangle\left\langle\tilde{\Psi}_{U}\right|+(1-\lambda) \frac{1}{2} \mathbb{1}_{2},
$$

where $\left|\tilde{\Psi}_{U}\right\rangle=\cos (\alpha)|\tilde{0}\rangle+\sin (\alpha)|\tilde{1}\rangle$ and $\lambda \equiv(2 q) /(1+q)$. Given $N$ states of the form (19), one can use the procedure described in 24 to purify the noisy state, i.e. to increase $\lambda$. For large $N$, the average fidelity - that is the overlap of the produced states with the state $\left|\tilde{\Psi}_{U}\right\rangle$ scales like $F \approx 1-\frac{1}{2 N} \frac{1-\lambda}{\lambda^{2}}$, whereas the yield -i.e. fraction of the number of produced states to the number of initial states $N$ - scales like $D \approx \lambda+\frac{1}{N} \frac{1-\lambda}{\lambda}$ [24. That is, for $N \rightarrow \infty$ one obtains almost perfect states $\left|\tilde{\Psi}_{U}\right\rangle$ with a yield $\lambda$. Note that the states $\left|\tilde{\Psi}_{U}\right\rangle$ are not uniformly distributed on the whole Bloch's sphere but rather only on the equatorial plane. Nevertheless, one can still use the same procedure as described in [24], where a uniform distribution was assumed. The corresponding values for $F$ and $D$ in our case are at least as big as the ones obtained in [24], since we have additional knowledge of the state, which may be used to further increase $F$ and $D$. Note that in order that purification is possible, we need that $\lambda>0$ and thus $q>0$. So all noisy gates of the form (15) and $U$ given by (16) can be purified if $q>0$.

To summarize, we managed to produce an arbitrary number of (almost) perfect states $\left|\tilde{\Psi}_{U}\right\rangle$ given several applications of the noisy operation $\mathcal{E}_{U}$. Note that $\left|\tilde{\Psi}_{U}\right\rangle$ can be transformed deterministically to $\left|\Psi_{U}\right\rangle$ by undoing the basis change (18). From the results of Sec. III, we know that the state $\left|\Psi_{U}\right\rangle$ can be used to implement $U$ with probability $p=1 / 2$. What remains is to show that one can implement $U$ with probability $p=1$. The simplest way to see this is the following: If we fail, we try to implement $U$ again, we make a third attempt and try to implement $U$ and so on. Every odd number of steps, say $2 j+1$, we stop the procedure if we have succeeded in $(j+1)$ steps and did not succeed in $j$ steps. In this case, we have applied the operation $U$ in total $(j+1)$ times and the operation $U^{\dagger} j$ times, which is equivalent to apply the operation $U$. This is a one-side bounded random walk with probability $p=1 / 2$, where one can easily see that the total success probability converges to $p=1$. Alternatively, one can also use the operation $U$ to prepare states $\left|\Psi_{U}\right\rangle$ with coefficient $2^{k} \alpha$, which is possible with probability $p=1 / 2^{2^{k}}$. These states can than be used to implement $U$ with $p=1$ following the procedure described in 20]. For a success probability $p=1-o(\epsilon)$, in total $o\left(\epsilon^{-1}\right)$ states $\left|\Psi_{U}\right\rangle$ with coefficient $\alpha$ are required.
Alternatively to the procedure described above, one may also use a method similar to the one of Sec. D to implement $U$ given several applications of $\mathcal{E}_{\mathcal{U}}$. By a sequence of measurements one first determines the state $E$, from which $\left|\Psi_{U}\right\rangle$ can be found and used to implement the operation $U$ (which is now known to $A$ ).

\section{TOMOGRAPHY OF OPERATIONS}

In this Section, we consider the problem of tomography of an arbitrary, unknown non-local CPM. Given several applications of the unknown CPM $\mathcal{E}$ and using the isomorphism (3), it is straightforward to completely determine the non-local CPM by a sequence of local measurements assisted by classical communication. To this aim, we use the operation $\mathcal{E}$ to prepare several copies of the associated state $E$ (3a). Now, using tomography for states [21], the state $E$ - and thus via (3b) also the CPM $\mathcal{E}$ - can be determined.

Next we show that a sequence of local measurements assisted by classical communication suffices to completely determine a non-local mixed state $E$ (and thus a nonlocal CPM $\mathcal{E}$ ). Let $A$ and $B$ be two spatially separated parties and $\left\{A_{i}\right\}\left[\left\{B_{j}\right\}\right]$ be an orthonormal [35] basis of self adjoint operators in $A[B]$. We have that $E_{A B}$ can be written as

$$
E_{A B}=\sum_{i, j} \lambda_{i j} A_{i} \otimes B_{j},
$$

where $\lambda_{i j}=\operatorname{tr}\left(A_{i} \otimes B_{j} E_{A B}\right)$ is the expectation value of the operator $A_{i} \otimes B_{j}$. Now, by measuring the operators $A_{i}\left[B_{j}\right]$ locally in $A[B]$ and using classical communication, one can establish the values of all $\lambda_{i j}$ and thus the state $E_{A B}$. In case the operation $\mathcal{E}$ acts on two qubits, the corresponding state $E_{A B}$ is a state of two four-level systems. The set of operators $\left\{A_{i}\right\}$ can e.g. chosen to be $\left\{\sigma_{i_{1}, i_{2}} \otimes \sigma_{i_{3}, i_{4}}\right\}$-where $\sigma_{i_{1}, i 2}$ are defined in Sec. III (see Eq. (6) ) - and similarly for $\left\{B_{i}\right\}$.

\section{PROBABILISTIC IMPLEMENTATION}

In this Section, we will show that the possibility to distinguish the state $\left|\Phi^{+}\right\rangle$from the other three Bell states and the capability to produce certain entangled states allows to implement probabilistically arbitrary two particle unitary operations. This has applications in the context of single photon experiments, since our method allows to implement two photon gates with a certain probability of success, which is already sufficient to implement entanglement distillation. Note that this should be feasible even with present day technology.

In the following, we concentrate on two qubit gates. Given the results of Sec. III, one observes that the possibility of creating certain entangled states, together 
with the capability of performing local Bell measurements allows to implement an arbitrary 2-qubit operation [36. That is, the problem to perform two-qubit gates is shifted to the problem of

\section{(i) creating certain entangled states and}

(ii) the capability to perform perfect Bell measurements.

In the following, we will discuss (i-ii) in the context of single photon experiments.

Regarding (i), in single photon experiments one is already able to create certain MES (e.g. via parametric down conversion). For example, MES of two qubits were created and used in teleportation experiments [37]. In addition, the creation of a three qubit GreenbergerHorne-Zeilinger (GHZ) state was reported [38]. Although non-linear elements are required in order to produce entangled states, it is much easier to use these elements in such a way that a known state is generated rather than using some non-linear elements to perform a controlled interaction between arbitrary states. Applying the isomorphism (3), one observes that the state $E$ corresponding to a general two-qubit unitary operation is a pure state of two four level systems (equivalently of four qubits). For example, the state corresponding to the CNOT operation [30] is given by $E_{\mathrm{CNOT}}=$ $\left(|00\rangle_{A}\left|\Phi^{+}\right\rangle_{B}+|11\rangle_{A}\left|\Psi^{+}\right\rangle_{B}\right) / \sqrt{2}$, while the SWAP operation (which is given by the mapping $|i j\rangle \rightarrow|j i\rangle$ ) is specified by $E_{\text {SWAP }}=\left|\Phi^{+}\right\rangle_{A_{1} B_{2}}\left|\Phi^{+}\right\rangle_{A_{2} B_{1}}$. Operations of the form (4) are specified by states of the form (5). Due to the fact that the states (5), as well as $E_{\mathrm{CNOT}}$ only have two Schmidt coefficients (when considered as a bipartite system $A-B)$, it should be possible to create them in the laboratory using present day technology.

What remains is (ii), the problem to perform Bell measurements. For single photons, using non-linear elements only (beam splitters and photo detectors), one is able to perform incomplete Bell measurements. In particular, one can perfectly distinguish the three sets of states $\left\{\left|\Phi^{+}\right\rangle\right\},\left\{\left|\Psi^{+}\right\rangle\right\}$, and $\left\{\left|\Phi^{-}\right\rangle,\left|\Psi^{-}\right\rangle\right\}$39. The optimality of this process using linear elements was discussed in 40]. Due to the fact that Bell measurements cannot be performed perfectly with linear elements [40] (see however 41]), it follows that two-qubit gates cannot be implemented with unit probability using the procedure described in Sec. III. However, even incomplete Bell measurements (which can already be performed in the laboratory) still allow for a probabilistic implementation of arbitrary two qubit gates. That is, with a certain probability the desired gate is applied, while otherwise a different (possibly unknown) operation is performed. In the latter case, the input state has to be discarded.

Let us investigate the consequences of incomplete Bell measurements a bit closer. From (3), we know that if both parties $A$ and $B$ obtain as a measurement outcome the state $\left|\Phi^{+}\right\rangle$, the desired operation was performed. Due to the fact that $\left|\Phi^{+}\right\rangle$can be perfectly distinguished from the other three Bell states using the methods described in
39,40, and all measurement outcomes are equal probable (in the case of two qubits, $p_{\Phi^{+}}=1 / 4$ ), this allows to implement the desired unitary operation with probability $p=1 / 16$. For unitary operations of the form (4), this probability can be further increased to $p=1 / 4$ given the fact that also $\left|\Psi^{+}\right\rangle$can be perfectly distinguished from the other Bell states. That is, if both party $A$ and $B$ find as a measurement outcome either $\left|\Phi^{+}\right\rangle$or $\left|\Psi^{+}\right\rangle$, the desired unitary operation was performed. In case the outcome was $\left|\Psi^{+}\right\rangle$, additional application of the local operation $\sigma_{x}$ is required (see Sec. III).

Note that probabilistic implementation of two-qubit gates is not useful in the context of quantum computation, as probabilistic operations may change the complexity class of the problem and may thus destroy the (exponential) speed up of the quantum algorithm in question. However, probabilistic gates are useful for processes such as entanglement distillation [4], which itself is already a probabilistic process. For example, this may help in the implementation of quantum repeaters [26] using photons only (i.e., for quantum communication over arbitrary distances). Due to the fact that photons are ideal candidates for quantum communication (due to their fast propagation), it is highly desirable to manipulate them directly (e.g. to perform entanglement purification as required in the quantum repeater protocol [26]) rather than mapping their states on the states of another physical system, e.g. of an ion or an atom, and vice versa. The method discussed in this section may help to achieve this task.

Recently, an alternative approach was presented by Pan et. al [42], where entanglement purification without CNOT operations was discussed. As this approach is concerned with a certain distillation procedure only, the solution provided in Ref. 42 to this specific problem is more efficient than the one we obtain here. However, we provide a more general framework which allows to implement arbitrary two-quibit operations probabilistically. Another proposal was presented by Knill et. al 41], who showed the implementation of a certain twoqubit operation with unit probability, taking full usage of all resources (i.e. using an arbitrary number of modes).

Note that similar techniques may be used to speed up -in some sense- slow two-particle interactions. The scenario we have in mind is the following: At a certain time - e.g. in course of a quantum computation - an entangling quantum operation should be performed on two particles. If the interaction between two particles is weak, the required interaction time in order that a entangling operation can be performed will be large. Now, instead of performing the operation when it is required, we use the (slow) interaction at some earlier stage to prepare certain entangled states. These states can then - at later timebe used to implement the two-particle operation almost immediately - once the two particles on which the operation should be performed arrive- using the procedure described in Sec. III. Although this procedure involves local Bell measurements, this will not slow down the process as for the implementation of those measurements, no 
two-particle interactions are required. For example, we can use internal levels of atoms or ions instead of local auxiliary qubits (see also Sec. IV]). Bell measurements in this case involve only single particle interactions between the different levels of a particle, which we assumed to be much faster than two-particle interactions.

\section{STORAGE AND COMPRESSION OF UNITARY OPERATIONS}

In this Section, we will discuss storage 43,20 and compression of unitary operations. We consider a (possibly infinite) set of unitary operations $U_{1}, U_{2}, \ldots, U_{N}$. Each operation is assigned an a priori probability $p_{i}$. We consider a long sequence of those operations, where each element $U_{i}$ of this sequence is chosen at random according to the probability distribution $\left\{p_{i}\right\}$. We are interested in the average number of qubits which are required to store one of the operations $U_{i}$ and implement the operations at later time with unit probability and high accuracy. We consider the following variations of this problem:

(i) The operations $U_{i}$ are local. That is, a party $A$ locally stores a certain number of qubits and uses these qubits to implement one of the local operation $U_{i}$ on some unknown state at later time. In this case, we are interested in the average number of qubits to be stored locally.

(ii) The operations $U_{i}$ are non-local. That is, two spatially separated parties $A$ and $B$ store a set of (possibly entangled) states and use these states later on to implement the non-local operation $U_{i}$. In this case, we allow the parties $A$ and $B$ to share some initial entangled states. The storage procedure, however, is restricted to local operations only. That is, parties $A$ and $B$ store (and compress) their part of the system individually. We are interested in the average number of qubits required in $A(B)$ to store one of the operations $U_{i}$ locally.

(iii) The operations $U_{i}$ are non-local. In contrast to (ii), one of the parties, say $A$, stores the operations $l o-$ cally. Using quantum communication, part of the stored system is then transferred to $B$ and finally used to implement the non-local operation $U_{i}$. In this case, we are interested in the average number of qubits which have to be stored locally in $A$ as well as in the required quantum communication, i.e. the average number of qubits which have to be transmitted from $A$ to $B$.

Note that in all cases, the unitary operation to be performed is at any stage unknown to $A$ (and $B$ ). We will show that storage of certain sets of unitary operations is possible. Furthermore, the scheme we propose allows to compress the amount of required storage qubits (as well as the amount of qubits transmitted from $A$ to $B$ in (iii)) if one restricts the set of allowed operations to a certain subset. It turns out that even for an infinite set of operations $U_{i}$, the average amount of required storage qubits per operation can be much smaller than one. These results can be viewed as an extension of the Schumacher data compression for states [6] to unitary operations. In fact, we will use the results of Ref. [6] to achieve this task.

Very recently, the problem of storage of a general unitary operation was considered by Vidal et al. [20] and an optimal solution was provided. In contrary to Ref. [20], we propose schemes which are capable to compress the required amount of storage qubits and also discuss storage of non-local operations. We will propose two different schemes for storage, one dealing with a possible infinite set of unitary operations $U_{i}$ and one with a finite set. We will discuss both schemes in the context of (i-iii).

\section{A. Local storage of local unitary operations}

We start out with (i), the local storage of a set of local unitary operations. We consider unitary operations acting on two qubits and assume that they are local, i.e., both qubits on which the operation should be performed are held by the same party, say $A$.

\section{Storage of an infinite set of unitary operations}

Here, we describe a procedure to store locally a unitary operation of the form $U(\alpha)$ (4) with arbitrary, unknown $\alpha$ using on average less than four [0.2257] qubits per operation if $0 \leq \alpha \leq \pi[\pi / 8]$. We assume uniform distribution of angles $\alpha$, i.e. any operation is equally likely.

We remind the reader that an operation $U(\alpha)$ for arbitrary $\alpha$ can be implemented by a sequence of operations of the form $U\left(\alpha_{k}\right)$ (4) with binary angles $\alpha_{k}=\pi / 2^{k}$ (see Sec. III (i-iii)). Using the fact that $\alpha$ can be written in binary notation (11) and assuming that all angles are equally likely, it follows that $n_{k}=0$ and $n_{k}=1$ are equal likely $\forall k$.

We first consider the implementation of $U\left(\alpha_{k}\right)$ for a certain $k \equiv N$ and $\alpha_{N}=\pi / 2^{N}$. Following the procedure described in Sec. III (ii), we have that if $n_{N}=1$, the following set of $N$ states is required to implement this operation with probability $p=1$ :

$$
G_{N}=\left\{\left|\Psi_{\alpha_{N}}\right\rangle,\left|\Psi_{2 \alpha_{N}}\right\rangle, \ldots,\left|\Psi_{2^{N-1} \alpha_{N}}\right\rangle\right\}
$$

where the corresponding probabilities are given by $p_{l}=$ $1 / 2^{l-1}$ for the $l^{\text {th }}$ state. If however $n_{N}=0$, no operation has to be performed. In this case, one can store the set of states $\tilde{G}_{N}=\left\{\left|\Psi_{0}\right\rangle,\left|\Psi_{0}\right\rangle, \ldots,\left|\Psi_{0}\right\rangle\right\}$, which corresponds to the implementation of the identity operation in each step. However, each step can be considered independently and involves with probability $p=1 / 2$ either the storage of the 
state $\left|\Psi_{2^{l} \alpha_{N}}\right\rangle$ for the $l^{\text {th }}$ step if $n_{N}=1$ or $\left|\Psi_{0}\right\rangle$ if $n_{N}=0$. Thus, one can us data compression of pure states [6] for each step independently. The corresponding compression factor $S_{j}$ for the $l^{\text {th }}$ step is given by the entropy of the operator $\tilde{\rho}$, which is an equal mixture of the state $\left|\Psi_{\alpha_{j}}\right\rangle$ and $\left|\Psi_{0}\right\rangle$, where $j \equiv(N-l)$. One finds

$$
S_{j}=-x_{j} \log _{2}\left(x_{j}\right)-\left(1-x_{j}\right) \log _{2}\left(1-x_{j}\right),
$$

with $x_{j}=\left(1+\cos \alpha_{j}\right) / 2$ and $\alpha_{j}=\pi / 2^{j}$. Recall also that the $l^{\text {th }}$ step has to be performed only with probability $p_{l}$. That is, the total amount of qubits required to store the operation $U\left(\alpha_{N}\right)$, where it is unknown whether it should be performed or not, is given by

$$
\sum_{l=1}^{N} S_{N-l} \frac{1}{2^{l-1}} .
$$

We now consider a sequence of operations of the form $U\left(\alpha_{k}\right)$ for $1 \leq k \leq \infty$, i.e. the implementation of $U(\alpha)$ with arbitrary $\alpha(0 \leq \alpha \leq \pi)$. Using (23), one finds that the total number of qubits needed to store one of those operations is on average given by

$$
\sum_{k=1}^{\infty} S_{k} \sum_{l=0}^{k-1} \frac{1}{2^{l}} \leq 2 \sum_{k=1}^{\infty} S_{k},
$$

which can evaluated to be 3.8942 . That is, less than 4 qubits per operation are required on average to store an arbitrary, unknown operations of the form $U(\alpha)$. In [20], it was found that on average two qubits suffice to store $U(\alpha)$.

However, if we restrict the possible values of $\alpha$ to $0 \leq \alpha \leq \pi / 8[\pi / 32]$, we find that the average amount of required storage qubits is given by 0.2257 [0.0206]. This can be seen by noting that in this case, the sum in Eq. (24) starts with $k=4[k=6]$ respectively. Thus we showed that unitary operations of the form $U(\alpha)$ can be stored locally, and that the average amount of qubits required for storage can be decreased if one restricts the operations to be stored. This result is similar to the one obtained by Schumacher [6] for the compression of a set of pure states.

\section{Compression of a finite set of unitary operations}

Here, we consider a finite set of unitary operations of the form $U\left(\alpha_{N}\right)$ (㓙), where $\alpha_{N}=\pi / 2^{N}$ and $1 \leq N \leq M$ and provide an alternative protocol for storage and compression. This set of operations can be viewed as the basic set required to implement arbitrary operations. We assume that each of the operation is equally likely. Again, we follow the procedure described in Sec. III (i-ii), in order to implement a certain operation of the form (4), say $U\left(\alpha_{N}\right)$, with unit probability. The set of states $G_{N}$ (21) is required, where the corresponding probabilities are given by $p_{k}=1 / 2^{k-1}$ for the $k^{\text {th }}$ state. Note that for different $N$, a different number of steps are required and thus a different number of states has to be stored. As this may cause problems, we fix the number of states to be stored for each operation to be $M$. In case less than $M$ steps are required, the state $\left|\Psi_{0}\right\rangle$ is stored in the remaining cases, which corresponds to the identity operation. Now, the implementation of any operation $U\left(\alpha_{N}\right)$ consists of at most $M$ steps, where in steps $(N+1), \ldots, M$ the identity operations is performed. The following table summarizes the states which are stored for each of the operations:

$$
\begin{aligned}
U\left(\alpha_{1}\right): G_{1} & =\left\{\left|\Psi_{\frac{\pi}{2}}\right\rangle,\left|\Psi_{0}\right\rangle,\left|\Psi_{0}\right\rangle,\left|\Psi_{0}\right\rangle, \ldots,\left|\Psi_{0}\right\rangle\right\} \\
U\left(\alpha_{2}\right): G_{2} & =\left\{\left|\Psi_{\frac{\pi}{4}}\right\rangle,\left|\Psi_{\frac{\pi}{2}}\right\rangle,\left|\Psi_{0}\right\rangle,\left|\Psi_{0}\right\rangle, \ldots,\left|\Psi_{0}\right\rangle\right\} \\
U\left(\alpha_{3}\right): G_{3} & =\left\{\left|\Psi_{\frac{\pi}{8}}\right\rangle,\left|\Psi_{\frac{\pi}{4}}\right\rangle,\left|\Psi_{\frac{\pi}{2}}\right\rangle,\left|\Psi_{0}\right\rangle, \ldots,\left|\Psi_{0}\right\rangle\right\} \\
\ldots & \\
U\left(\alpha_{M}\right): G_{M} & =\left\{\left|\Psi_{\alpha_{M}}\right\rangle,\left|\Psi_{2 \alpha_{M}}\right\rangle,\left|\Psi_{4 \alpha_{M}}\right\rangle, \ldots,\left|\Psi_{\frac{\pi}{2}}\right\rangle\right\} .
\end{aligned}
$$

Recall that the $k^{\text {th }}$ state is always used in the $k^{\text {th }}$ step. We denote by $C_{k}$ the $k^{\text {th }}$ column, which consists of the $k^{\text {th }}$ element of each $G_{l}$. As the columns $C_{k}$ correspond to the different steps, we have that column $k$ is only required with probability $p=1 / 2^{k-1}$ and all steps - and thus all columns $C_{k}$ - can be treated independently. That is, we store each of the columns $C_{k}$ independently. Due to the fact that all states within each column $C_{k}$ are equal likely and non-orthogonal, one can use data compression [6]. The compression factor $S_{k}$ for column $C_{k}$ is given by the entropy of the density operator $\rho_{k}$, where $\rho_{k}$ is an equal mixture of all the states of column $k$, and column $k$ is only required with probability $p=1 / 2^{k-1}$. Thus the total number of qubits required to store one of the operations $U\left(\alpha_{N}\right), 0 \leq N \leq M$ is given by $\sum_{k=1}^{M} S_{k} / 2^{k-1}$. For example, for $M=100$ [1000] we obtain an average amount of 0.245 [0.0361] qubits which have to be stored on average to implement one of the 100 [1000] operations picked at random.

\section{B. Storage of non-local unitary operations in $A$ and}

B

Here, we consider (ii), the storage of a set of non-local unitary operations. We will discuss variations of both protocols described in the previous section, taking into account that we now have two spatially separated parties and the operations are non-local. That is, the states to be stored are entangled states and we consider local storage of the subsystem belonging to $A(B)$. This means that both, the coding and decoding procedure has to be local, but may be assisted by classical communication.

We first consider the storage of an infinite set of unitary operations of the form $U(\alpha)$ (see Sec. VII A 1). We follow the same protocol as described in Sec. VII A 1, however we now use a different kind of data compression. The protocol described in Sec. VII A 1 involves storage of two 
equal likely states, $\left|\Psi_{0}\right\rangle$ or $\left|\Psi_{\alpha_{N}}\right\rangle$ for some $\alpha_{N}=\pi / 2^{N}$. Note that the state $\left|\Psi_{\alpha_{N}}\right\rangle$ is an entangled state, so in contrast to the previous section, we cannot use standard data compression for pure states, as we are restricted to local operations only. However - as shown in Appendix A - it is also possible to achieve local data compression for a set of entangled states. That is, each of the parties manipulates only its own subsystem and can thereby reduce the average number of qubits required to store its part of the entangled state, without affecting the entanglement with the other system. Note that this problem is equivalent to the data compression of mixed states with commuting density operators, where the entanglement with some other system should be preserved. It turns out that the compression factor for $A(B)$ is given by the entropy of an operator $\tilde{\rho}$, which is an equal mixture of the reduced density operators $\rho_{A}^{i}\left(\rho_{B}^{i}\right)$ corresponding to the states $\left|\Psi_{0}\right\rangle,\left|\Psi_{\alpha_{N}}\right\rangle$. Note that this corresponds to the upper bound on the number of qubits to be stored in case the entanglement with another system is not required to be preserved [44]. While it is known that this is not the optimal compression rate in case the entanglement with some other system is not required to be preserved, it is not clear whether the compression rate is already optimal under this stronger restriction. In our specific case, we obtain

$$
S_{N}=-x_{N} \log _{2}\left(x_{N}\right)-\left(1-x_{N}\right) \log _{2}\left(1-x_{N}\right),
$$

with $x_{N}=\left(1+\cos ^{2} \alpha_{N}\right) / 2$. Using now this local compression protocol instead of Schumacher's for pure states in the protocol of Sec. VII A 1, one finds that the the average number of qubits which have to be stored locally in $A(B)$ is given by 4.7758 if $0 \leq \alpha \leq \pi$. If we restrict the possible values of $\alpha$ to $0 \leq \alpha \leq \pi / 8$ [ $\pi / 32]$, we find that the average amount of required storage qubits is reduced to 0.3976 [0.0379].

Regarding the storage of a finite set of unitary operations (see Sec. VII A 2), we follow the same protocol as described in Sec. VIIA2 and use again a different kind of data compression due to the fact that we are restricted to local operations. This time, data compression for a finite set $\left\{\left|\Psi_{i}\right\rangle\right\}$ of $M$ entangled states is required. The entangled states are all equal likely and are of the form $\left|\Psi_{\alpha_{N}}\right\rangle$. It turns out (see Appendix A) that one can achieve a compression rate which is given by the entropy of a density operator $\tilde{\rho}$, which is defined as an equal mixture of the reduced density operators $\rho_{A}^{i}$ corresponding to the state $\left|\Psi_{i}\right\rangle$. One finds that the total number of qubits required on average to store one of the operations $U\left(\alpha_{N}\right), 0 \leq N \leq M$ locally in $A(B)$ is given by 0.333 [0.050] qubits for $M=100$ [1000].

\section{Storage of non-local unitary operations in $A$}

Finally, we consider (iii) the local storage of a nonlocal unitary operation in $A$. That is, we consider a local memory (in $A$ only), but we want to implement the operation non-locally. It turns out that this problem is a trivial combination of the previous two problems. We have that one can use the methods described in Sec. VII A to store the operations locally in $A$, and one obtains the the same compression rates. The average amount of quantum communication from $A$ to $B$-which is required to implement the operation non-locally - can be found using the method described in Sec. VIIB. That is, one part of the entangled system is compressed and send through a quantum channel to $B$. The compression rate can be calculated in a similar way as in Sec. VIIB, however one has to take into account that the state $\left|\Psi_{\pi / 2}\right\rangle$ is a separable state and thus no quantum communication is required to transmit one part of this state. For example, one finds in case of an infinite set of operations of the form $U(\alpha)$ with $0 \leq \alpha \leq \pi[\pi / 8]$ that the required amount of quantum communication from $A$ to $B$ is given by 2.7758 [0.3976] qubits.

This last method clearly distinguishes between the required amount of local storage qubits and the non-local content of the operation, i.e. the average amount of quantum communication. Note that storing the operations locally (see Sec. VII A) requires a smaller amount of storage qubits than storing a non-local operation directly in $A$ and $B$ (see Sec. VIIB).

\section{NON-LOCAL MEASUREMENTS}

In this Section, we consider the implementation of nonlocal measurements. We consider two spatially separated parties, $A$ and $B$, each possessing a $d$-level system. The two parties want to perform a complete, joint measurement on their system, specified by a set of rank $n_{k}$ Projectors $\left\{P_{k}\right\}$ such that $\sum_{k} P_{k}^{A B}=\mathbb{1}_{A} \otimes \mathbb{1}_{B}$. The questions we pose are the following: How can the parties implement this non-local measurement? What are the entanglement properties of those measurements; that is, (i) what is the amount of entanglement required to implement a certain measurement? (ii) what is the average amount of entanglement which can be produced given a single application of the non-local measurement?

We provide several procedures to implement arbitrary non-local von Neumann measurements and discuss their entanglement properties. We show that the required amount of entanglement depends on the measurement to be implemented. We introduce examples of nonlocal measurements which can be implemented using less than one ebit of entanglement. One can easily generalize some of our results to implement also arbitrary measurement, described by a positive operator valued measure (POVM), i.e. a set of positive operators $O_{k}^{A B}$ such that $\sum_{k} O_{k}^{A B}=\mathbb{1}_{A} \otimes \mathbb{1}_{B}$.

First, we note that the amount of entanglement required to implement the non-local measurement depends on (i) whether one is only interested in the measurement 
outcome or (ii) the system should in addition be in the corresponding state after the measurement. For example, one can perform a complete Bell measurement (i.e. a measurement in the basis (6)) on a state of two qubits using one ebit of entanglement regarding (i), while two ebits are required in case of (ii).

Proposal 1: A trivial procedure to perform an arbitrary bipartite measurement is the following: The state of system $B$ is teleported to $A$, consuming $\log _{2}(d)$ ebits. Then, the measurement is performed locally in $A$, which already suffices in case of (i). Regarding (ii), one also has to teleport the particle back to $B$, consuming again $\log _{2}(d)$ ebits. Note that in case of a complete Bell measurement, i.e. a measurement in the basis (6), where each basis state is a MES, this procedure is in fact optimal. On the one hand, one consumes two ebits to implement the measurement. On the other hand, one can also obtain an average amount of two ebits given a single application of a non-local Bell measurement. One just has to consider the operator $E_{i}$ (3) associated to each possible outcome of the Bell measurement. One observes that the nonlocal entanglement of all $E_{i}$ is given by two ebits, and each measurement outcome is equal likely. This leads to an average amount of entanglement of two ebits. We have that if the amount of entanglement required to implement an operation, $E_{\mathrm{im}}$ equals the amount of entanglement which can be obtained given a single application of the operation, $E_{\mathrm{cr}}$, the first process is optimal and $E_{\text {im }}$ is the minimal amount of entanglement required to implement the operation. This is due to the fact that $E_{\text {cr }} \leq E_{\text {im }}$, otherwise one could create entanglement for free. However, if one wants to measure the joint system $A, B$ in a basis which is not maximally entangled, one might expect that the required amount of entanglement is smaller than $2 \log _{2}(d)$ ebits. With the following method, we show that this is indeed the case.

Proposal 2: We consider the situation were all $P_{k}$ are rank one, i.e. $n_{k}=1$ and thus $P_{k}=\left|\phi_{k}\right\rangle_{A B}\left\langle\phi_{k}\right|$. We define a non-local unitary operation $U$ by

$$
U=\sum_{k=1}^{d^{2}}|k\rangle_{A B}\left\langle\phi_{k}\right|,
$$

where $|k\rangle_{A B}=\left|a_{k}\right\rangle_{A}\left|b_{k}\right\rangle_{B}$, and $\left\{\left|a_{i}\right\rangle\right\}\left[\left\{\left|b_{i}\right\rangle\right\}\right]$ is some local basis in $A[B]$ respectively, with $1 \leq k \leq d$. The procedure takes place as follows: First, the parties apply the non-local unitary operation $U$, using e.g. the procedure described in Sec. III for $d=2$, consuming an amount of entanglement which is specified by the operation $U$. In case $U$ is only weakly entangling, e.g. if $\left\langle k \mid \phi_{k}\right\rangle \approx 1$ (i.e. $\left|\phi_{k}\right\rangle$ are only weakly entangled states), the required entanglement is small (see Sec. III) [45]. Then, parties $A$ and $B$ both perform a local measurement specified by projectors on the states $\left\{\left|a_{i}\right\rangle\right\}\left[\left\{\left|b_{i}\right\rangle\right\}\right]$ respectively and communicate the outcome of the measurement classically. In case they obtained the outcome $a_{k}, b_{k}$, they know that the outcome of the measurement is $k$, i.e. they measured the projector $P_{k}$. Concerning (i), the procedure ends at this point. Regarding (ii), $A$ and $B$ in addition implement the operation $U^{\dagger}$ to ensure that the system is also in the required state after the measurement. Alternatively, they could also prepare the measured system in state $\left|\phi_{k}\right\rangle$, as due to the implementation of the measurement, any possible entanglement with some auxiliary system is destroyed anyway. We note that the choice of the local basis in $A,\left\{\left|a_{i}\right\rangle\right\}$ and $B,\left\{\left|b_{i}\right\rangle\right\}$ is not fixed and may also change the entanglement properties of the operation $U$. This can be seen by considering the following trivial example: We have $d=2$ and $\left|\phi_{00}\right\rangle=$ $|00\rangle,\left|\phi_{01}\right\rangle=|01\rangle,\left|\phi_{10}\right\rangle=|10\rangle$ and $\left|\phi_{11}\right\rangle=|11\rangle$. By choosing $\left|a_{1}\right\rangle\left|b_{1}\right\rangle=|0\rangle$ and $\left|a_{2}\right\rangle\left|b_{2}\right\rangle=|1\rangle$, we have that $U=\mathbb{1}_{A B}$, i.e. no entanglement is required to perform the measurement. If we however choose the mapping $\left|\phi_{00}\right\rangle \rightarrow|00\rangle,\left|\phi_{01}\right\rangle \rightarrow|10\rangle,\left|\phi_{10}\right\rangle \rightarrow|01\rangle,\left|\phi_{11}\right\rangle \rightarrow|11\rangle$, we find that the operation $U=U_{\text {swap }}$, which requires two ebits to implement [16]. In this case, the choice of the proper local basis is trivial, however we do not know the optimal choice for a general measurement. Note also that this procedure fails to implement non-local measurements where the rank of some projector $P_{k}$ is larger than one. For example, if $P_{1}=|00\rangle_{A B}\langle 00|$ and $P_{2}=\mathbb{1}_{A B}-P_{1}$, this procedure fails to project in the subspace spanned by $P_{2}$, as it already gives a fine-graining within this subspace, which is a different problem. The next method will overcome this limitation.

Proposal 3: Here, we consider a complete set of $M$ non-local projectors $P_{k}$ which might have arbitrary rank $n_{k}$. Clearly, $\sum_{k=1}^{M} n_{k}=d^{2}$. Alice uses an $M$-level auxiliary system initially prepared in state $|1\rangle$, which is used to label all possible measurement outcomes. We define a unitary operation $U$ acting on the auxiliary system $\tilde{A}$ and the joint system $A B$ as follows:

$$
\begin{aligned}
U= & \sum_{j=1}^{M}\left[\left(|j\rangle_{\tilde{A}}\langle 1|+| 1\rangle_{\tilde{A}}\langle j|\right) \otimes P_{j}^{A B}\right. \\
& \left.+\left(\mathbb{1}_{\tilde{A}}-|1\rangle_{\tilde{A}}\langle 1|-| j\rangle_{\tilde{A}}\langle j|\right) \otimes P_{j}^{A B}\right] .
\end{aligned}
$$

After application of $U$, the auxiliary system $\tilde{A}$ is measured in the basis $\{|j\rangle\}$. If the outcome $k$ is found, one readily observes that this corresponds to measuring the projector $P_{k}$ on the system $A B$. Note that no further operations are required, as the system $A B$ is already in the appropriate state (ii). The amount of entanglement required to implement the non-local measurement is again specified by the operation $U$.

For example, if $d=2$ and $P_{1}=(|00\rangle\langle 00|+| 11\rangle\langle 11|)$, $P_{2}=(|01\rangle\langle 01|+| 10\rangle\langle 10|)$, it turns out that one can create one ebit given a single measurement of this kind. To see this, we prepare the system $A B$ in the product state $\rho=1 / 2\left(\left|\Phi^{+}\right\rangle\left\langle\Phi^{+}|+| \Psi^{+}\right\rangle\left\langle\Psi^{+}\right|\right)$and perform the measurement. In case we obtain outcome "1" ["2"], the state after the measurement is $\left|\Phi^{+}\right\rangle\left[\left|\Psi^{+}\right\rangle\right]$respectively. In both cases, we created one ebit. However, it is not clear whether one ebit of entanglement also suffices to implement the corresponding unitary operations 
$U=\mathbb{1}^{\tilde{A}} \otimes P_{1}^{A B}+\sigma_{\tilde{A}}^{\tilde{A}} \otimes P_{2}^{A B}$. Although the state $E_{U}$ associated to $U$ via (3) has an amount of entanglement of one ebit and $U=U^{\dagger}$, it is not clear whether a single copy of the state $E_{U}$ suffices to implement $U$.

It would be interesting to establish the minimal amount of entanglement required to implement a general, non-local measurement.

\section{MULTIPARTY OPERATIONS}

In this Section, we generalize some of the previous results to multiparty systems. We consider several spatially separated systems $A, B, \ldots, Z$, each possessing several $d$-level systems. We first generalize the isomorphism (3) between $\mathrm{CPM} \mathcal{E}$ and positive operators $E$ to multiparty systems. Here, $\mathcal{E}$ acts on several $d$-level systems, one located in each site $A, B, \ldots, Z$ and $E$ is a positive operator on the Hilbert space $\mathcal{H}_{A_{1,2}} \otimes \ldots \otimes \mathcal{H}_{Z_{1,2}}$. We have that $\mathcal{H}_{A_{i}}=\mathbb{C}^{d}$ and similar for the remaining parties. For a $N$-party system, it is easy to show that

$$
\begin{aligned}
E_{A_{1,2} \ldots Z_{1,2}}= & \mathcal{E}\left(P_{A_{1,2}} \otimes \ldots \otimes P_{Z_{1,2}}\right) \\
\mathcal{E}\left(\rho_{A_{1} \ldots Z_{1}}\right)= & d^{2 N_{\operatorname{tr}}} \operatorname{tr}_{A_{2,3} \ldots Z_{2,3}} \\
& \left(E_{A_{1,2} \ldots Z_{1,2}} \rho_{A_{3} \ldots Z_{3}} P_{A_{2,3}} \ldots P_{Z_{2,3}}\right) .
\end{aligned}
$$

The interpretation is similar to the one of Eq. (3). On one hand, (29a) states that $E$ can be created from a $N$ party product state, where each party prepares locally a MES. On the other hand, (29b) tells us that given $E$ (particles $\left.A_{1,2} B_{1,2} \ldots Z_{1,2}\right)$, one can implement the multiparticle operation $\mathcal{E}$ on an arbitrary state $\rho$ of $N d$-level systems (particles $A_{3} B_{3} \ldots Z_{3}$ ) by measuring locally the projector $P$ (2) on particles $A_{2,3}, B_{2,3}, \ldots, Z_{2,3}$ in each of the locations. Note that the probability of success is given by $p=1 / d^{2 N}$.

As in the bipartite case, one may ask for a certain map $\mathcal{E}$ whether it is capable to create entanglement. Since for multiparty systems, there exist many different kinds of entanglement (see e.g. 46 49]), one may also ask which kind of entanglement can be produced. Again, all these questions can be answered by establishing the entanglement properties of the operator $E$ associated to the $\mathrm{CPM} \mathcal{E}$ via (29a). In particular, if $E$ is bound entangled [48], then $\mathcal{E}$ can only create BES. In a similar way, given some BES one can easily construct the corresponding map which is capable of generating BES of the same kind.

One may also consider the implementation of arbitrary $N$-qubit operations with unit probability. On one hand, any $N$-qubit operation can be written as a sequence of bipartite CNOT operations and single qubit unitary operations, for which we already established a protocol. On the other hand, we may consider $N$-qubit unitary operations of a specific form and show directly how to implement them with unit probability given certain states. We consider a unitary operation of the form

$$
U_{N}\left(\alpha_{M}\right)=e^{-i \alpha_{M} \sigma_{x}^{A_{1}} \otimes \ldots \otimes \sigma_{x}^{Z_{1}}},
$$

where $\alpha_{M}=\pi / 2^{M}$. It turns out that a natural extension of the protocol of Sec. III (i-iii) allows to implement operations of the form (30) with probability $p=1$. The operator associated with the unitary operation $U_{N}\left(\alpha_{M}\right)$ is given by $E_{A_{1,2}, \ldots, Z_{1,2}}=\left|\psi_{\alpha_{M}}\right\rangle\left\langle\psi_{\alpha_{M}}\right|$, where

$$
\begin{aligned}
\left|\psi_{\alpha_{M}}\right\rangle= & \cos \left(\alpha_{M}\right)\left|\Phi^{+}\right\rangle_{A_{1,2}}\left|\Phi^{+}\right\rangle_{B_{1,2}} \ldots\left|\Phi^{+}\right\rangle_{Z_{1,2}} \\
& -i \sin \left(\alpha_{N}\right)\left|\Psi^{+}\right\rangle_{A_{1,2}}\left|\Psi^{+}\right\rangle_{B_{1,2}} \ldots\left|\Psi^{+}\right\rangle_{Z_{1,2}}
\end{aligned}
$$

Regarding (i), we just note that Bell measurements and the corresponding local unitary operations are performed at all location $A, B, \ldots, Z$. For all possible measurement outcomes, it is easy to observe that the operation performed on some state $\rho_{A_{1}, \ldots, Z_{1}}$ will either be (i) $U\left(\alpha_{M}\right)$ or (ii) $U\left(-\alpha_{M}\right)$, each possibility appearing with probability $p=1 / 2$. Steps (ii) and (iii) can be adopted without changes, which finally allows to implement an operation of the form (30) with arbitrary angle $\alpha$ and unit probability. Note that operations (30) are capable to create GHZ-like entanglement and are thus truly $N$-qubit entangling operations.

\section{SUMMARY}

To summarize, we have provided several applications of an previously introduced isomorphism between operations an states. First, we discussed how to use this isomorphism to establish separability and entangling properties of operations $\mathcal{E}$ and to construct physical operations which are capable to create bound entangled states. In addition, we showed how to implement an arbitrary non-local two-qubit operation consuming an amount of entanglement which is proportional to the entangling capability of the operation.

Then, we have shown how to implement several techniques developed for states - such as purification or data compression - also for operations. In particular, we have shown that a known, noisy, non-local unitary operation as well as an unknown, noisy, local unitary operation can be purified. In a similar way, we use these results to establish tomography of arbitrary operations. Then, we showed that unitary operations can be stored locally and non-locally and that the amount of required qubits for storage can be decreased, which can be viewed as generalization of data compression to unitary operations. In this context, we also provided a protocol which allows for local data compression of a set of entangled states. Note that it is straightforward to obtain a number of other results which were developed for states also for operations. For example, it is easy to show that also unitary operations can be cloned (via cloning of the corresponding state $E$ ) or teleported (via teleportation of the states required to store the operation) $[1920$. In case of cloning, 
one has to take into account that the cloned states allow for a probabilistic, imperfect implementation of the required operation only.

We also provided a method to implement arbitrary two-photon gates probabilistically with present day technology, which opens the way for practical quantum communication over arbitrary distances. Finally, we discussed the implementation of non-local measurements and generalized some of our results to multi-party systems.

\section{ACKNOWLEDGEMENTS}

We thank B. Kraus, G. Giedke and G. Vidal for interesting discussions. This work was supported by the Austrian SF under the SFB "control and measurement of coherent quantum systems" (Project 11), the European Community under the TMR network ERBFMRX-CT96-0087 and project EQUIP (contract IST1999-11053), the ESF, and the Institute for Quantum Information $\mathrm{GmbH}$.

\section{APPENDIX A: LOCAL DATA COMPRESSION FOR A SET OF ENTANGLED STATES}

In this appendix, we consider the problem of local data compression of a set of pure, entangled states, where all reduced density operators commute. Note that this problem is equivalent to the problem of data compression of a set of commuting mixed states under the restriction that entanglement with some other systems should be preserved. Let $G=\left\{\left|\Psi_{i}\right\rangle\right\}_{i=1}^{L}$ be a set of $L$ pure states, where

$$
\left|\Psi_{i}\right\rangle=c_{\alpha_{i}}|00\rangle_{A B}+s_{\alpha_{i}}|11\rangle_{A B}
$$

and $c_{\alpha_{i}} \equiv \cos \left(\alpha_{i}\right), s_{\alpha_{i}} \equiv \sin \left(\alpha_{i}\right)$. Each state is assigned a prior probability $p_{i}$. Two spatially separated parties $A$ and $B$ are fed an unending sequence of states $\left|\Psi_{j}\right\rangle$, where each successive state is chosen randomly and independently from the set $G$ according to the probability distribution $\left\{p_{i}\right\}$. A sequence of length $N$ is of the form

$$
\left|\Psi_{i_{1} i_{2} \ldots i_{N}}\right\rangle=\left|\Psi_{i_{1}}\right\rangle\left|\Psi_{i_{2}}\right\rangle \ldots\left|\Psi_{i_{N}}\right\rangle
$$

and appears with probability $p_{i_{1} i_{2} \ldots i_{N}}=p_{i_{1}} p_{i_{2}} \ldots p_{i_{N}}$. The parties $A$ and $B$ want to store the sequences locally, i.e they are allowed to perform local operations and classical communication. We are interested in the average amount of qubits per signal state which are required in $A(B)$ to store the signals faithfully. We will use as a criterium the so-called GLOBAL-FID criterium 44]; that is we require that the average global fidelity of all possible sequences is $1-\epsilon$. Note that we consider the so called "blind case" [44, that is neither $A$ nor $B$ know the specific sequence (33).
Let $\rho_{i}^{A}=\operatorname{tr}_{B}\left(\left|\Psi_{i}\right\rangle\left\langle\Psi_{i}\right|\right)$ be the reduced density operator of system $A$ of the state $\left|\Psi_{i}\right\rangle$ and

$$
\tilde{\rho}^{A}=\sum_{i=1}^{L} p_{i} \rho_{i}^{A}
$$

be the weighted average of the reduced density operators of our signal source. We denote by $S\left(\tilde{\rho}^{A}\right)=$ $\operatorname{tr}\left(\tilde{\rho}^{A} \log _{2} \tilde{\rho}^{A}\right)$ be the von Neumann entropy of $\tilde{\rho}^{A}$.

Given a sequence of length $N, N$ sufficiently large, we provide a protocol with the following properties:

(i) The required amount of storage qubits in $A(B)$ is given by $N S\left(\tilde{\rho}^{A}\right)+\delta$.

(ii) The average global fidelity (averaged over all possible sequences) $\bar{F}$ is given by $1-\epsilon$.

We have that $\delta$ is some function which is of the form $\delta=$ $\mu N^{\beta}$ for some $\mu>1,1 / 2<\beta<1$ and $\epsilon \rightarrow 0$ as $N \rightarrow \infty$. That is, on average $S\left(\tilde{\rho}^{A}\right)$ qubits per signal state have to be stored locally in $A(B)$. Note that we do not claim that this the optimal compression rate achievable.

For pedagogical reasons, we will proof our statement in the simplest case, where the set $G$ consists of two pure states only. We will even assume that $\left|\Psi_{1}\right\rangle=|00\rangle$ and $\left|\Psi_{2}\right\rangle=c_{\alpha}|00\rangle+s_{\alpha}|11\rangle$ and $p_{1}=p_{2}=1 / 2$. Note that the proof can be easily generalized to an arbitrary number of signal states and an arbitrary probability distribution.

We have that

$$
\tilde{\rho}^{A}=\frac{1+c_{\alpha}^{2}}{2}|0\rangle\left\langle 0\left|+\frac{1-c_{\alpha}^{2}}{2}\right| 1\right\rangle\langle 1| .
$$

We define local projectors $P_{A}\left(P_{B}\right)$ acting on $N$ qubits as follows:

$$
P_{A}=P_{B}=\sum_{k=k^{-}}^{k^{+}} P_{k}
$$

where $k^{ \pm}=\left(1+c_{\alpha}^{2}\right) / 2 \pm \mu N^{\beta}, \mu>0,1 / 2<\beta<1$ and

$$
P_{k}=\sum_{\text {perm }}|0\rangle\left\langle\left. 0\right|^{\otimes k} \otimes \mid 1\right\rangle\left\langle\left. 1\right|^{\otimes N-k} .\right.
$$

The sum in (37) runs over all possible $b_{N, k} \equiv N ! /[k !(N-$ $k)$ !] permutations (without repetitions) of $k$ zeros and $N-k$ ones. Thus $P_{k}$ is a projector in the subspace spanned by all states which contain exactly $k$ zeros and $(N-k)$ ones. The dimension of $P_{k}$ is given by $b_{N, k}$.

The projector $P_{A}\left(P_{B}\right)$ is measured locally in $A(B)$. If the measurement is successful, $\log _{2}(d)$-where $d=$ $\left.\operatorname{dim}\left(P_{A}\right)\right)$ - qubits are used to store the resulting state in $A$. This can be accomplished by relabeling the states which span $P_{A}$ to $\{|l\rangle\}_{l=1}^{d}$ and store those states locally, which clearly requires $\log _{2}(d)$ qubits. The decoding procedure consists of undoing the relabeling. In case the measurement is not successful, some state $\left|0_{E}\right\rangle$ is stored instead. We show that: (i) $\log _{2}(d)=N S(\tilde{\rho})+\delta$ 
and (ii) $\bar{F}=\sum_{i_{1} i_{2} \ldots i_{N}} p_{i_{1} i_{2} \ldots i_{N}} F_{i_{1} i_{2} \ldots i_{N}}>1-\epsilon$, where $F_{i_{1} i_{2} \ldots i_{N}}=\left|\left\langle\Psi_{i_{1} i_{2} \ldots i_{N}}\left|P_{A} \otimes P_{B}\right| \Psi_{i_{1} i_{2} \ldots i_{N}}\right\rangle\right|^{2}$ and the sum runs over all possible sequences. That is, the storage procedure requires the announced amount of qubits and the average fidelity is sufficiently large.

Regarding (i), it is easy to see that $d=\operatorname{dim}\left(P_{A}\right) \leq$ $\left(k^{-}+k^{+}+1\right) b_{N, k_{0}}$, where $k_{0} \in\left[k^{-}, k^{+}\right]$is the value that maximizes $b_{N, k}$ in this interval. Substituting the values of $k^{-}, k^{+}$in this bound, we find that $\log _{2}(d)=N S\left(\tilde{\rho}^{A}\right)+\delta$ as required.

We now concentrate on (ii), the average global fidelity $\bar{F}$. Consider a sequence of the form (33) which contains $j$ states $\left|\Psi_{1}\right\rangle$ and $(N-j)$ states $\left|\Psi_{2}\right\rangle$ (i.e. the number of $i_{k}$ which are equal to one is given by $j$ ). We denote such a sequence by $|\Psi(j)\rangle$. Note that there are $b_{N, j}$ sequences of this kind. For all those sequences, we find

$$
\begin{aligned}
F_{j} & =\left|\left\langle\Psi(j)\left|P_{A} \otimes P_{B}\right| \Psi(j)\right\rangle\right|^{2} \\
& =\left|\sum_{k ; k^{-} \leq(j+k) \leq k^{+}} c_{\alpha}^{2 k} s_{\alpha}^{2(N-k)} b_{N-j, k}\right|^{2},
\end{aligned}
$$

The average fidelity $\bar{F}$ is given by

$$
\bar{F}=\frac{1}{2^{N}} \sum_{k^{-} \leq j \leq k^{+}} b_{N, j} F_{j},
$$

where $2^{-N}$ is the probability that a certain sequence appears, $b_{N, j}$ is the number of sequences of the form $|\Psi(j)\rangle$ and $F_{j}$ is given in (38). We have that

$$
\bar{F} \geq \sum_{j=j^{-}}^{j^{+}} \frac{1}{2^{j}} \frac{1}{2^{N-j}} b_{N, j} F_{j},
$$

where $j^{ \pm}=N / 2 \pm \mu / 3 N^{\beta}$. In this case, one can also bound $F_{j}$ and finds

$$
F_{j} \geq\left|\sum_{k=\tilde{k}^{-}}^{\tilde{k}^{+}} c_{\alpha}^{2 k} s_{\alpha}^{2(N-k)} b_{N-j, k}\right|^{2}
$$

where $\tilde{k}^{ \pm}=c_{\alpha}^{2}(N-j) \pm \mu / 3 N^{\beta}$ and we have that $k^{-} \leq(j+k) \leq k^{+}$as required. By noting that a binomial distribution is asymptotically equivalent to a normal (Gaussian) distribution, the fidelity $F_{j} \forall j$ can be seen to be bounded from below by $\Phi\left(2 \mu N^{\beta-1 / 2}\right)$, where $\Phi(x) \equiv 1 / \sqrt{2 \pi} \int_{-x}^{x} e^{y^{2} / 2} d y$. For our choice of $\mu, \beta$, we have that $F_{j} \rightarrow 1$ when $N \rightarrow \infty$. In a similar way, one shows that also $\bar{F} \rightarrow 1$ when $N \rightarrow \infty$, as after bounding $F_{j}$ as stated above, (40) also corresponds to a binomial distribution centered at $j=N / 2$. This finishes the proof of the statements (i-ii).

In a similar way, one can carry out the analysis for a set of $L$ entangled states and an arbitrary probability distribution $\left\{p_{i}\right\}$. In this case, $k^{ \pm}=N \sum_{i=1}^{L} p_{i} c_{\alpha_{i}}^{2} \pm \mu N^{\beta}$ and some of the binomial distributions are replaced by multinomial distributions. Also in this case, one finds that $\bar{F} \rightarrow 1$ for $N \rightarrow \infty$ and that the dimension of the projector $P_{A}\left(P_{B}\right)$ is given by the $N$ times the entropy of the operator $\tilde{\rho}^{A}(34)$.

1] J. I. Cirac, W. Dür, B. Kraus and M. Lewenstein, quantph/0007057 (to appear in Phys. Rev. Lett.)

[2] C. H. Bennett, G. Brassard, C. Crépeau, R. Jozsa, A. Peres, and W. K. Wootters, Phys. Rev. Lett. 70, 1895 (1993).

[3] C. H. Bennett, H. J. Bernstein, S. Popescu and B. Schumacher, Phys. Rev. A 53, 2046(1996)

[4] C. H. Bennett, G. Brassard, S. Popescu, B. Schumacher, J. A. Smolin and W. K. Wootters, Phys. Rev. Lett. 76, 722 (1996); C. H.Bennett, D. P. DiVincenzo, J. A. Smolin and W. K. Wootters, Phys. Rev. A 54, 3824 (1996).

[5] D. Deutsch, A. Ekert, C. Macchiavello, S. Popescu, and A. Sanpera, Phys. Rev. Lett. 77 , 2818 (1996).

[6] B. Schumacher, Phys. Rev. A 51, 2738-2747 (1995)

[7] V. Bužek and M. Hillery, Phys. Rev. A 54, 1844 (1996); V. Bužek et al. , Phys. Rev. A 56, 3446 (1997); N. Gisin and S. Massar, Phys. Rev. Lett. 79, 2153 (1997). D. Bruss et al. , Phys. Rev. A 57, 2368 (1998); R. Werner, Phys. Rev. A 58, 1827 (1998); P.Zanardi, ibid. 58, 3484 (1998); D. Bruss, A. Ekert, and C. Macchiavello, Phys. Rev. Lett. 81, 2598 (1998).

[8] K. Vogel and H. Risken, Phys. Rev. A 40, R2847 (1989).

[9] P. Zanardi, C. Zalka and L. Faoro, Phys. Rev. A 62, 30301R (2000)

[10] W. Dür, G. Vidal, J. I. Cirac, N. Linden and S. Popescu, quant-ph/0006034

[11] B. Kraus and J. I. Cirac, quant-ph 0011050

[12] P. Zanardi, quant-ph/0010074

[13] D. Gottesman, quant-ph/9807006.

[14] A. Chefles, C. R. Gilson and S. M. Barnett, quantph/0003062 and quant-ph/0006106.

[15] J. Eisert, K. Jacobs, P. Papadopoulos and M. B. Plenio, Phys. Rev. A 62052317 (2000).

[16] D. Collins, N. Linden and S. Popescu, quant-ph/0005102

[17] for a review see M., P. and R. Horodecki, in "Quantum Information - basic concepts and experiments", in print (Springer, Berlin 2000); M. Lewenstein, D. Bruß, J. I. Cirac, B. Kraus, M. Kus, J. Samsonowicz, A. Sanpera and R. Tarrach, quant-ph/0006064

[18] J. I. Cirac, A. K. Ekert, S. F. Huelga, and C. Macchiavello, Phys. Rev. A. 59, 4249 (1999)

[19] S. F. Huelga, J. A. Vaccaro, A. Chefles and M. B. Plenio, quant-ph/0005061

[20] G. Vidal and J. I. Cirac, quant-ph/0012067

[21] J. F. Poyatos, J. I. Cirac and P. Zoller, Phys. Rev. Lett. 78, 390 (1997); I. L. Chuang and M. A. Nielsen, J. Mod. Opt. 44, 2455 (1997)

[22] A. Acín, E. Jané and G. Vidal, quant-ph/0012015; G. M. D'Ariano and P. Lo Presti, quant-ph/0012071

[23] A. Jamiolkowski, Rep. of Math. Phy. No. 4, 3275 (1972)

[24] J. I. Cirac, A. K. Ekert and C. Macchiavello, Phys. Rev. 
Lett. 82, 4344 (1999).

[25] If $\rho$ is a multiqubit state and $U$ is performed on qubits one and two, the noisy operation is defined as follows: $\mathcal{E}(\rho)=q U_{12} \rho U_{12}^{\dagger}+\frac{1-q}{16} \operatorname{tr}_{12}\{\rho\} \otimes \mathbf{1}_{12}$, i.e. the state of the remaining particles is described by their reduced density operator. This noise model was also used in the context of quantum repeaters 26.

[26] H.-J. Briegel, W. Dür, J. I. Cirac, and P. Zoller, Phys. Rev. Lett. 81, 5932 (1998); W. Dür, H.-J. Briegel, J. I. Cirac, and P. Zoller, Phys. Rev. A 59, 169-181 (1999).

[27] W. Dür, J. I. Cirac, M. Lewenstein and D. Bruß, Phys. Rev. A 61, 062313 (2000);

[28] D. P. DiVincenzo, P. W. Shor, J. A. Smolin, B. M. Terhal and A. V. Thapliyal, Phys. Rev. A 61, 062312 (2000)

[29] M. Horodecki and P. Horodecki, Phys. Rev. A. 59, 4206 (1999).

[30] The CNOT operation is defined by the following mapping of states, written in the standard basis: $|i\rangle_{A}|j\rangle_{B} \rightarrow$ $|i\rangle_{A}|i \oplus j\rangle_{B}$, where $\oplus$ denotes addition modulo 2 .

[31] The 4-qubit state $\rho_{\mathcal{E}}$ can in an appropriate basis be written as $q\left|\psi^{-}\right\rangle_{A B}\left\langle\psi^{-}\right|+(1-q) / 16 \mathbf{1}_{4}$. One can easily check that the partial transposition of $\rho_{\mathcal{E}}$ is non positive iff $q>1 / 9$. Projecting the state $\rho_{\mathcal{E}}$ locally — both in a $A$ and $B$ - in the subspace spanned by $\{|0\rangle,|1\rangle\}$, the resulting state is of Werner form [32] and can be distilled to singlet form if $q>1 / 9$ [ [- Due to the fact that non positive partial transposition is a necessary condition for distillability, we have that $\rho_{\mathcal{E}}$ is distillable to singlet form if and only if $q>1 / 9$.

[32] R. F. Werner, Phys. Rev. A 40, 4277 (1989).

[33] C. A. Sackett et al., Nature 404, 256-259 (2000); A. Rauschenbeutel et al., Science 288, 2024-2028 (2000)

[34] G. Giedke, H.J. Briegel, J.I. Cirac, and P. Zoller, Phys. Rev. A 59, 2641 (1999).

[35] We are considering Hilbert space of operators with scalar product $\left(A_{i}, A_{k}\right)=\operatorname{tr}\left(A_{i}^{\dagger} A_{k}\right)$. Two operators $A_{i}$ and $A_{k}$ are orthonormal if $\left(A_{i}, A_{k}\right)=\delta_{i, k}$.

[36] D. Gottesman and I. L. Chuang, quant-ph/9908010

[37] D. Bouwmeester et al., Nature 390, 575 (1997); D. Boschi et al., Phys. Rev. Lett. 80, 1121 (1998).

[38] D. M. Greenberger, M. Horne, A. Zeilinger, Bell's theorem, Quantum Theory, and Conceptions of the Universe, ed. M. Kafatos, Kluwer, Dordrecht 69 (1989); D. Bouwmeester et al., Phys. Rev. Lett. 82 , 1345 (1999). J.- W. Pan et al., Nature 403, 515 (2000)

[39] H. Weinfurter, Europhys. Lett. 25, 559 (1994); S. L. Braunstein and A. Mann, Phys. Rev. A 51, R1727 (1995); K. Mattle, H. Weinfurter and A. Zeilinger, Phys. Rev. A 53, R1209 (1996)

[40] N. Lütkenhaus, J. Calsamiglia and K.-A. Suominen, Phys Rev. A 59, 3295 (1999); J. Calsamiglia and N. Lütkenhaus, quant-ph/0007058

[41] E. Knill, R. Laflamme and G. Milburn, quant$\mathrm{ph} / 0006088$

[42] J.- W. Pan, C. Simon, C. Bruckner and A. Zeilinger, quant-ph/0012026

[43] M. A. Nielsen and I. L. Chuang, Phys. Rev. Lett. 79, 321 (1997).

[44] H. Barnum, C. M. Caves, C. A. Fuchs, R. Jozsa and B. Schumacher, quant-ph/0008024
[45] For example, in the case of two qubits and $\left|\phi_{i j}\right\rangle=$ $U^{\dagger}(\alpha)|i j\rangle_{A B}$ with $U(\alpha)$ given in (4) and $\alpha \ll 1$, we have that the states $\left|\phi_{i j}\right\rangle$ are weakly entangled. We can chose $U=U(\alpha)$ in our procedure to implement the non-local measurement, and obtain that the required amount of entanglement to implement $U$ is given by $\bar{E} \approx 6 \alpha$ ebits (see Sec. III), which can be much smaller than one. Regarding (i), the measurement can be implemented using $6 \alpha$ ebits, while in case of (ii) less than $12 \alpha$ ebist are required.

[46] W. Dür, G. Vidal and J. I. Cirac, Phys. Rev. A 62, 062314 (2000)

[47] W. Dür and J. I. Cirac, quant-ph 0011025; W. Dür, J. I. Cirac, and R. Tarrach, Phys. Rev. Lett. 83, 3562 (1999); W. Dür and J. I. Cirac, Phys. Rev. A 61, 042314 (2000).

[48] W. Dür and J. I. Cirac, Phys. Rev. A 62, 022302 (2000).

[49] A. Thapliyal, Phys. Rev. A 59 (1999) 3336; J. Kempe, Phys. Rev. A 60 (1999) 910; C. H. Bennett, D. P. DiVincenzo, T. Mor, P. W. Shor, J. A. Smolin, and B. M. Terhal, Phys. Rev. Lett. 83, 3081 (1999); D. P. DiVincenzo, T. Mor, P. W. Shor, J. A. Smolin, B. M. Terhal, quant-ph/9908070; J. A. Smolin, quant-ph/0001001; P. W. Shor, J. A. Smolin and A. V. Thapliyal, quant$\mathrm{ph} / 0005117$; 